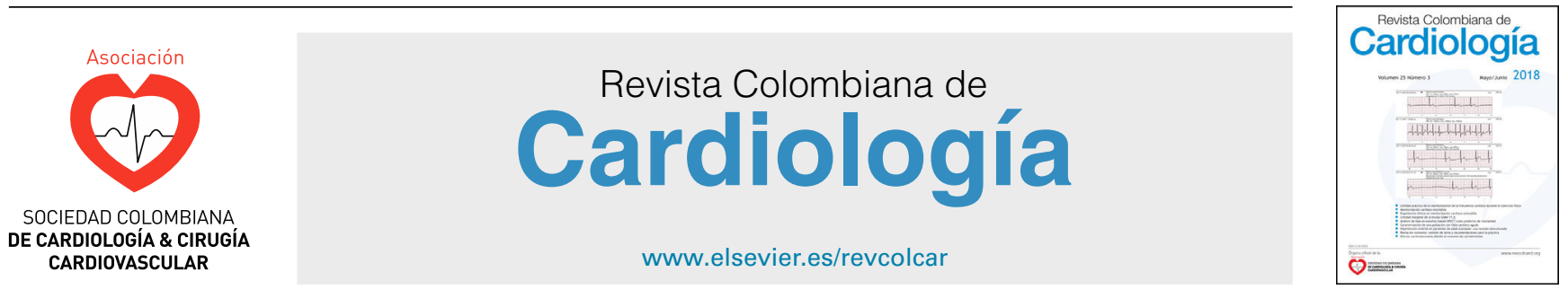

CARDIOLOGÍA DEL ADULTO - REVISIÓN DE TEMAS

\title{
Variabilidad de la frecuencia cardiaca como factor predictor de las enfermedades cardiovasculares
}

\section{Laura Veloza ${ }^{a}$, Camilo Jiménez ${ }^{a}$, Daniel Quiñones ${ }^{a}$, Felipe Polanía ${ }^{a}$, Lida C. Pachón-Valero ${ }^{\mathrm{b}}$ y Claudia Y. Rodríguez-Triviño ${ }^{\mathrm{b}, *}$}

\author{
a Semillero Fisiología Cardiovascular, Facultad de Ciencias de la Salud, Fundación Universitaria Navarra - Uninavarra, Neiva, \\ Colombia \\ b Departamento de Ciencias fisiológicas, Facultad de Ciencias de la Salud, Fundación Universitaria Navarra - Uninavarra, Neiva, \\ Colombia
}

Recibido el 24 de marzo de 2018; aceptado el 2 de enero de 2019

Disponible en Internet el 19 de junio de 2019

\section{PALABRAS CLAVE \\ Variabilidad de la frecuencia cardiaca ;Enfermedades cardiovasculares; Sistema nervioso autónomo}

\section{KEYWORDS}

Heart rate variability; Cardiovascular diseases; Autonomous nervous system

\begin{abstract}
Resumen La variabilidad de la frecuencia cardiaca se conoce como la variación en el tiempo que transcurre entre los intervalos RR del electrocardiograma y refleja la actividad del sistema nervioso autónomo sobre la función cardiaca. Su aumento se considera un factor protector para el corazón y su medición podría ser una herramienta predictiva temprana o diagnóstica en enfermedades cardiovasculares. El sistema nervioso autónomo genera efectos inotrópicos y cronotrópicos en la función cardiaca, que pueden aumentar o disminuir esta variabilidad. Existen diversos métodos de medición de la variabilidad de la frecuencia cardiaca; el más común es el Holter seguido por el sistema POLAR, además se han desarrollado programas de software clínico (Kubios ${ }^{\oplus}$, Sinus Core ${ }^{\oplus}$ ) que han demostrado validez en estas mediciones. La variabilidad de la frecuencia cardiaca puede emplearse como factor predictor en la aparición de eventos coronarios, accidentes cerebrovasculares y muerte súbita, entre otros.

(c) 2019 Sociedad Colombiana de Cardiología y Cirugía Cardiovascular. Publicado por Elsevier España, S.L.U. Este es un artículo Open Access bajo la licencia CC BY-NC-ND (http:// creativecommons.org/licenses/by-nc-nd/4.0/).
\end{abstract}

Heart rate variability as a predictive factor of cardiovascular diseases

Abstract Heart rate variability is defined as the variation in time between the RR intervals of the electrocardiogram, and reflects the activity of the autonomous nervous system over cardiac function. Its increase is considered as a protective factor for the heart, and its measurement could be used as a tool for the early prediction or diagnosis of cardiovascular diseases. The autonomous nervous system generates inotropic and chronotropic effects on cardiac function,

\footnotetext{
* Autor para correspondencia.

Correos electrónicos: coor.fisiologia@uninavarra.edu.co, clausr3@gmail.com (C.Y. Rodríguez-Triviño).
} 
which can increase or decrease this variability. There are several methods for measuring heart rate variability. The most common is the Holter device, followed by the Polar system. Clinical computer programs have also been developed (Kubios ${ }^{\circledast}$, Sinus Core ${ }^{\circledast}$ ) that have shown to be valid in the measurements. Heart rate variability may be used as a predictive factor for the appearance of coronary events, including among others, cerebrovascular accidents and sudden death.

(C) 2019 Sociedad Colombiana de Cardiología y Cirugía Cardiovascular. Published by Elsevier España, S.L.U. This is an open access article under the CC BY-NC-ND license (http:// creativecommons.org/licenses/by-nc-nd/4.0/).

\section{Introducción}

La variabilidad de la frecuencia cardiaca es la variación en el tiempo que transcurre en milésimas de segundos entre los intervalos $\mathrm{R}$ y $\mathrm{R}$ medidos en un electrocardiograma, $\mathrm{y}$ demuestra la interacción entre el sistema nervioso autónomo y la frecuencia cardiaca (fig. 1) ${ }^{1,2}$. El aumento o la disminución de la variabilidad de la frecuencia cardiaca están relacionados con la respuesta del sistema simpático y parasimpático; en otras palabras, la variación de tiempo en milisegundos que se da entre latido y latido está producida por la interacción del sistema nervioso autónomo con el sistema cardiovascular ${ }^{2,3}$. Como está bien documentado, en estado de reposo hay un predominio parasimpático importante que mantiene una perfusión ventricular izquierda adecuada y favorece la ausencia de microisquemias, hecho que también se asocia con distensibilidad aórtica apropiada y baja resistencia vascular sistémica ${ }^{4,5}$.

\section{Relación de la variabilidad de la frecuencia cardiaca y las enfermedades cardiovasculares}

Las enfermedades cardiovasculares son un conjunto de afecciones que involucran el corazón y los vasos sanguíneos y existen diferentes factores de riesgo que determinan la predisposición a padecerlas ${ }^{6}$. Las más prevalentes son la cardiopatía coronaria, asociada a aterosclerosis ${ }^{7}$ y la

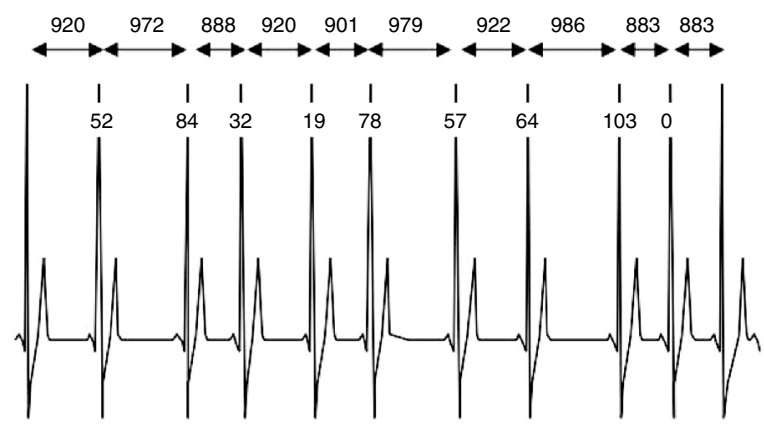

Figura 1 Ejemplo de un trazado de ECG con más de once complejos QRS. Se muestran los tiempos de intervalo R-R y la diferencia entre los intervalos R-R adyacentes. Fuente: Achten et al., 2003 (5). enfermedad cerebrovascular. Estas han incrementado en un 41\% en las últimas dos décadas, dando así lugar a 17.3 millones de muertes al año ${ }^{8}$. Adicionalmente, sobresalen las cardiopatías congénitas, que tienen una incidencia de $8 / 1.000$ nacidos vivos por año ${ }^{9}$ y la trombosis venosa profunda en miembros inferiores con una incidencia de 1 a 2 por 1.000 personas $^{10}$.

La Organización Panamericana de Salud (OPS) junto con la Organización Mundial de la Salud (OMS), realizaron un estudio en 2013 sobre la morbilidad y mortalidad presentes en el continente americano, en el que se concluyó que las enfermedades cardiovasculares fueron las de mayor prevalencia, con un $37,6 \%$ y dentro de estas las miocardiopatías y las enfermedades cerebrovasculares fueron las más frecuentes. En Colombia y la región Surcolombiana el comportamiento es similar según los informes periódicos del Departamento Administrativo Nacional de Estadística (DANE) y el boletín epidemiológico de la Secretaría de salud departamental del Huila ${ }^{11,12}$.

De otra parte, los factores de riesgo para enfermedades cardiovasculares se dividen en modificables y no modificables. En los primeros se incluyen: hipertensión arterial, obesidad, sedentarismo, dislipidemia, entre otros ${ }^{12-15}$. En tal sentido, la hipertensión arterial (HTA) se mantiene como uno de los principales factores de riesgo modificables para desarrollar enfermedad cerebrovascular ${ }^{16,17}$ según la Guía de Hipertensión de la Asociación americana del corazón $2017^{18,19}$. Esta se encuentra en un $49 \%$ de los casos de falla cardiaca y en un $35 \%$ del total de eventos cardiovasculares ateroscleróticos ${ }^{20,21}$. Por otro lado, el sedentarismo es responsable del aumento de enfermedades metabólicas, cardiovasculares y degenerativas. La actividad física es una de las principales recomendaciones para la prevención de enfermedades como la hipertensión, ya que un ejercicio físico de intensidad moderada contribuye a su disminución y aumenta los parámetros de variabilidad de la frecuencia cardiaca $^{22}$.

Entre los factores de riesgo no modificables, figuran edad, sexo, lipoproteína -A y factores genéticos ${ }^{23}$. A medida que pasa el tiempo el sistema parasimpático pierde dominio sobre el sistema cardiovascular, lo cual se asocia con mayores frecuencias cardiacas y disminución de la variabilidad de la frecuencia cardiaca, acompañada de menor distensibilidad aórtica y perfusión coronaria disminuida ${ }^{13}$. En las mujeres premenopáusicas la producción de estrógenos como 
el estradiol tiene un papel cardioprotector al inhibir las catecolaminas, fenómeno que disminuye la frecuencia cardiaca y la presión arterial; por consiguiente, tienen menor probabilidad de desarrollar enfermedades cardiovasculares respecto a las mujeres postmenopáusicas y a los hombres, quienes se relacionan con bajas cantidades de estrógenos y generalmente una variabilidad de la frecuencia cardiaca más baja y frecuencias cardiacas más altas ${ }^{24}$. Adicionalmente, mayores descargas de adrenalina y noradrenalina relacionada con la testosterona se asocian con variabilidad de la frecuencia cardiaca más baja ${ }^{13}$. Finalmente, entre los factores genéticos se halla la diabetes mellitus tipo I por neuropatías diabéticas que generan un aumento del tono simpático de un 20 a un 40\%, y dan origen a hipertensión y variabilidad de la frecuencia cardiaca disminuida ${ }^{25,26}$.

El diagnóstico temprano de las enfermedades cardiovasculares es decisivo para disminuir la mortalidad y la morbilidad, así como la identificación de factores de riesgo que pueden alertar de manera más oportuna. Se han utilizado algunos biomarcadores para determinar la correlación de la insuficiencia cardíaca con la fracción de eyección preservada o reducida; los más relevantes son el péptido natriurético tipo $B$ pro $\mathrm{N}$-terminal o péptido natriurético cerebral, la troponina $\mathrm{T}$ o I de alta sensibilidad, la proteína C-reactiva, el índice de albúmina urinaria-creatinina, la relación entre renina y aldosterona, el dímero $D$, el fibrinógeno, el supresor soluble de tumorigenicidad, la galectina-3, la cistatina $C$, el inhibidor del activador del plasminógeno y la interleucina 6; sin embargo, la variabilidad de la frecuencia cardiaca se ha utilizado solo como parámetro investigativo y podría también emplearse como parámetro de diagnóstico temprano, si se desarrollan estudios poblacionales que apoyen su uso clínico ${ }^{27-29}$.

\section{Efecto del sistema nervioso autónomo en el sistema cardiovascular que impacta en la variabilidad de la frecuencia cardiaca}

Existen varios factores que inciden sobre la variabilidad de la frecuencia cardiaca y en general sobre el inotropismo y el cronotropismo cardiaco; tal es el caso del sistema nervioso autónomo (SNA), que modifica factores cardiovasculares como la frecuencia cardiaca, la fuerza contráctil, el redireccionamiento del flujo sanguíneo a zonas de mayor demanda vascular y el control de la presión arterial a corto plazo a través de diferentes receptores tanto simpáticos como parasimpáticos $^{30}$. La estimulación simpática en general debería aumentar la frecuencia cardiaca y disminuir la variabilidad de la misma; no obstante, esta modulación no es lineal, ya que se ha visto que la frecuencia cardiaca tiene influencia poderosa en el valor clínico de la variabilidad de la misma. Pese a ello, esta influencia puede ser modificada efectivamente por una simple manipulación matemática, dejando a la variabilidad de la frecuencia cardiaca como predictor de eventos no $\operatorname{cardiacos}^{31}$. Dicha estimulación simpática de receptores adrenérgicos $\beta 1, \beta 2$ y $\beta 3$ que por lo general son más sensibles a la adrenalina y un poco menos a la noradrenalina, genera aumentos de la concentración de adenosín monofosfato cíclico intracelular ( $\uparrow$ [AMPc]i) que concluye en diferentes efectos; los $\beta 1$ en el corazón aumentan el gasto cardíaco ya que activan la proteína $G$ heterotrimérica Gs y estimulan a la adenilato-ciclasa, lo cual antagoniza los efectos de los receptores muscarínicos y aumenta el $\mathrm{Ca}^{++}$intracelular en el citosol, principalmente a través de proteína quinasa dependiente del cAMP, que desencadena la fosforilación de los canales de $\mathrm{Ca}_{2}{ }^{++}$tipo $L$ dependientes de voltaje $\left(I_{C a++}\right)$, e incrementa así la respuesta de $\mathrm{Ca}_{2}{ }^{++}$inducido por $\mathrm{Ca}_{2}{ }^{++}$y las corrientes If del nódulo sinusal. Adicionalmente, eleva la velocidad de repolarización a través de la salida activa de potasio de los canales rectificados de potasio $I_{K s}$ e $I_{K r}$, así como también la fosforilación de la proteína reguladora fosfolamban, con el aumento consecuente de la efectividad de la acción de la bomba sarcolémica de calcio (SERCA1). Esta influencia está presente en la etapa cuatro de potencial de acción, la cual se acorta y genera disminución de la etapa lenta de la despolarización diastólica espontánea (DDE) de las células excitables no contráctiles del sistema eléctrico cardiaco $^{32-34}$. Algunas sustancias antagonistas $\beta 1$ son antihipertensivos de utilidad; los $\beta 2$ están en concentraciones altas en el músculo bronquial y los $\beta 3$ en los adipocitos.

Los receptores $\alpha 1$ predominan en los vasos sanguíneos y los $\alpha 2$ en los terminales presinápticos, y son más sensibles a la noradrenalina que a la noradrenalina. La estimulación de los receptores $\alpha 1$ adrenérgicos genera estimulación de la proteína $G$ y efectos de las subunidades $\alpha$ activadas, que a su vez estimulan la gosfolipasa C; esta enzima convierte el PIP2 en inositol trifosfato (IP3) y diacilglicerol (DAG), y ambos generan vasocontricción vascular secundaria al estímulo de receptores de IP3 en el retículo sarcoplasmático que permiten la salida efectiva de $\mathrm{Ca}_{2}{ }^{++}$, mientras que el DAG activa la fosfoquinasa C (PKC). Los medicamentos agonistas $\alpha 1$ son eficaces como descongestivos nasales y los antagonistas $\alpha 2$ se han usado para tratar la impotencia. La médula suprarrenal también tiene influencia sobre el inotropismo y cronotropismo ya que es una adaptación especial de la división simpática, homóloga a la neurona simpática posganglionar que libera adrenalina al torrente sanguíneo. Este componente neuroendocrino de la salida simpática potencia la capacidad de la división simpática para difundir su mensaje y disminuye la variabilidad de la frecuencia cardiaca.

EI SNP a diferencia del SNS disminuye la frecuencia cardiaca y bloquea los efectos simpáticos sobre el corazón ${ }^{34}$. La acetilcolina ( $\mathrm{ACh}$ ) es un neurotransmisor segregado por ramas del nervio vago, el cual estimula los receptores muscarínicos $M 1, M 2, M 3$ y $M 5$ sensibles a $A C h$, que a su vez están acoplados a proteínas Gs. La estimulación de los $M 2$ cardiacos bloqua la acción simpática disminuyendo la concentracion de [AMPC] i y adicionalmente estimula la salida de potasio ocasionando repolarización a través de receptores rectificados de potasio dependientes de acetilcolina $\left(\mathrm{IK}_{A C h}\right)$, que son compatibles con acciones inotrópicas y cronotrópicas negativas, que tienden a aumentar el tiempo de la variabilidad de la frecuencia cardiaca ya que incrementan el tiempo de despolarización lenta en la etapa cuatro del potencial de acción de respuesta lenta. La predominancia del parasimpático en estado de reposo favorece la irrigación 
coronaria ya que permite que la diástole sea más prolongada y que el ventrículo izquierdo esté sometido a periodos más cortos de hipoxia ${ }^{32,35}$.

\section{Medición de la variabilidad de la frecuencia cardiaca}

La frecuencia cardiaca y variabilidad de la frecuencia cardiaca son inversamente proporcionales, además en el análisis de esta variación pueden influir distintos factores como edad, género, temperatura, hora del día, estado de actividad (activo o en reposo), carga de trabajo, consumo de alcohol o tabaco, entre muchos otros ${ }^{36}$. Para medir la variabilidad de la frecuencia cardiaca se utilizan distintos métodos; entre ellos el más común es el electrocardiograma de 24 horas. Esta técnica muestra gráficamente cada una de las ondas $\mathrm{R}$ que se generan con cada latido, permitiendo el análisis del tiempo en milisegundos que hay entre los intervalos R-R y las pequeñas variaciones que se pueden detectar entre intervalos consecutivos. Se lleva a cabo mediante un monitor Holter que registra el ritmo cardíaco de la persona durante 24 horas mientras esta realiza sus actividades habituales ${ }^{36}$. Otra forma de medir la variabilidad de la frecuencia cardiaca es mediante los aparatos portátiles POLAR, generalmente usados por deportistas, ya que permiten cuantificar los intervalos $\mathrm{R}-\mathrm{R}$ mientras la persona practica ejercicio físico ${ }^{36}$. Este sistema también se utiliza en personas sometidas a ambientes especiales, como pilotos, astronautas y deportistas náuticos. Con la ayuda de algunas técnicas como exponer a un paciente a estrés físico o administración de inotrópicos, se pueden hacer mediciones de laboratorio cortas de 2-5 minutos que permiten comparar la variabilidad de la frecuencia cardiaca de una persona antes y después de exponerlo a los estimuladores del sistema nervioso autónomo ${ }^{36}$. En la actualidad se han utilizado diversos software en estudios de variabilidad de la frecuencia cardiaca, como el programa Kubios y SinusCor, que ofrecen el análisis de dominio de tiempo y frecuencia, y han demostrado tener buena correlación en condiciones de reposo y actividad; además, sus interpretaciones están relacionadas con el pronóstico clínico y la información de diagnóstico. Los parámetros estadísticos y geométricos, la transformación rápida de Fourier y los diagramas periódicos basados en auto-regresión son enfoques utilizados comúnmente para la evaluación de señales de intervalos RR en estado de reposo ${ }^{37-39}$. Los parámetros que se verifican están divididos en dos grandes grupos: métodos en el dominio del tiempo, que incluyen los métodos estadísticos y geométricos, y métodos en el dominio de frecuencia. Los parámetros para el análisis de dominio de tiempo según las Guías de práctica clínica de la Sociedad Española de Cardiología en la monitorización ambulatoria del electrocardiograma y presión arterial son: intervalo RR promedio $>900 \mathrm{~ms}$, desviación estándar de todos los intervalos RR (SDNN) de $141 \pm 39 \mathrm{~ms}$, desviación estándar de la media de los intervalos RR medidos cada 5 min durante todo el registro (SDANN) de $127 \pm$ $35 \mathrm{~ms}$, raíz cuadrada de la media de la suma de las diferencias entre intervalos RR elevados al cuadrado (RMSSD) de 27 $\pm 12 \mathrm{~ms}$, RR50 dividido por el número total de intervalos RR (PNN50\%) es del 3\%. Los métodos geométricos incluyen el índice triangular de variabilidad de la frecuencia cardiaca o el número total de intervalos RR dividido por la altura del histograma de todos los intervalos RR medidos en una escala discreta con bins de $78.125 \mathrm{~ms}$ (índice SDRR) $37 \pm 15 \mathrm{~ms}$. Las medidas en el dominio de la frecuencia, aunque relevantes desde el punto de vista investigativo, usualmente no aparecen en el reporte del Holter tradicional, así que tienen uso clínico restringido ${ }^{29}$.

La variabilidad de la frecuencia cardiaca tiene distintos usos, entre los cuales está evaluar la función autonómica cardiaca. Existen pocas evidencias acerca de la relación que hay entre la variabilidad de la frecuencia cardiaca y la enfermedad cardiovascular; sin embargo, la disminución de esa variabilidad se ha asociado con distintas enfermedades como hipertensión arterial, diabetes, hipercolesterolemia y malos hábitos de vida como el sedentarismo y el consumo de $\operatorname{tabaco}^{40,41}$.

\section{Aplicaciones de la variabilidad de la frecuencia cardiaca en la práctica clínica}

El análisis de la frecuencia cardiaca se ha estudiado desde hace varias décadas. Se ha encontrado una asociación con la enfermedad cardiovascular en hombres y mujeres, que fue independiente de los factores de riesgo cardiovascular asociados. Los eventos coronarios también aumentaron cuando la frecuencia cardiaca previa fue más elevada ${ }^{13}$. Se considera la variabilidad de la frecuencia cardiaca como una herramienta de evaluación de las condiciones cardiacas autónomas capaz de predecir el riesgo cardiovascular. Algunos valores de corte sugeridos para determinar el riesgo, establecen parámetros que según los resultados pueden dar un riesgo alto, moderado o bajo: Intervalo $\mathrm{RR}<750 \mathrm{~ms}$ riesgo alto, $\mathrm{RR} 750-900 \mathrm{~ms}$ riesgo moderado, RR. > $900 \mathrm{~ms}$ riesgo bajo, SDRR < $50 \mathrm{~ms}$ riesgo alto, SDRR 50-100 ms riesgo moderado, SDRR $>100 \mathrm{~ms}$ $\mathrm{R}$ riesgo bajo, $\mathrm{pRR} 50<3 \% \mathrm{R}$ riesgo alto, $\mathrm{pRR} 50>3 \% \mathrm{R}$ riesgo bajo, SDARR $<8 \mathrm{~ms}$ riesgo alto, SDARR $8-12 \mathrm{~ms}$ riesgo moderado, SDARR $>12 \mathrm{~ms}$ riesgo bajo, índice SDRR $<25 \mathrm{~ms}$ riesgo alto, índice $S D R R=25-40 \mathrm{~ms} R$ riesgo moderado, índice SDRR $>40 \mathrm{~ms} R$ riesgo bajo $^{28,42}$.

El análisis de la variabilidad de la frecuencia cardiaca ha llevado a algunos investigadores a asociarla con accidente cerebrovascular isquémico y a concluir que es contraria al grosor del músculo liso de las arterias coronarias dado que la distensibilidad arterial es inversamente proporcional a la presión que se genera en los vasos ${ }^{40}$. Mediante relaciones como estas, claramente se pueden establecer pronósticos y tratamientos ante problemas cardiovasculares. La medición de la variabilidad de la frecuencia cardiaca también ha sido usada por los profesionales en el ámbito deportivo, ya que ayuda a evaluar el estado de salud de los deportistas durante el entrenamiento, y de este modo les permite fijar variaciones en el ejercicio según la adaptación que tienen a la actividad física ${ }^{40,43,44}$. Otros estudios han demostrado que la eliminación del impacto de la frecuencia cardiaca sobre la variabilidad de la frecuencia cardiaca hace que la variabilidad de la frecuencia cardiaca sea más predictiva de muerte no cardíaca. Sin embargo, el cálculo de este impacto hace que la variabilidad de la frecuencia cardiaca sea un mejor predictor de mortalidad cardiovascular ${ }^{25}$. 
En el estudio de Sacha et al., se hicieron modificaciones matemáticas a fin de observar su influencia sobre la repetibilidad en los análisis de la variabilidad de la frecuencia cardiaca ${ }^{31}$. En este participaron 24 pacientes (14 hombres y 10 mujeres) a quienes se les registró la frecuencia cardiaca dos veces al día a la misma hora, mañana y noche, durante 30 días; se obtuvieron 1.121 muestras y 512 intervalos RR. Dentro de los resultados se pudo observar cómo la frecuencia cardiaca no solo genera una ayuda en el poder predictivo de la variabilidad de la frecuencia cardiaca, sino también su influencia en la repetibilidad ${ }^{31}$.

El uso de variabilidad de la frecuencia cardiaca para predecir muerte súbita ha venido relegándose dada la dificultad del proceso en el campo clínico, pese a lo cual en los Estados Unidos se estima que al año se presen$\tan \mathbf{4 0 0 . 0 0 0}$ fallecimientos debido a muerte súbita. Una de las medidas profilácticas adoptadas para esta situación es la implantación del cardiodesfibrilador o cardioversor, que lamentablemente es una opción costosa y con riesgos en la implantación. La evaluación de la variabilidad de la frecuencia cardiaca permite identificar pacientes con bajo riesgo cardiaco en quienes no sea necesario el uso de cardiodesfibrilador ya que la variabilidad de la frecuencia cardiaca actúa como un medio pronóstico para aquellos con miocardiopatía dilatada no isquémica ${ }^{45}$. Rashba et al. hicieron un seguimiento para estudiar la morbilidad y mortalidad de dos grupos de pacientes con falla cardiaca $(n=458)$, en uno de los cuales administraron medicamentos clásicos como tratamiento y en otro, adicional a los medicamentos, usaron el cardiodesfibrilador. Se observó que los pacientes con cardiopatía dilatada no isquémica que tenían estable la SDNN tenían mejor pronóstico y no requerían implante de cardiodesfibrilador, mientras que la disminución de la SDNN se asoció con mayor mortalidad ${ }^{45}$.

El estudio de Yadav RL et al. en el departamento de fisiología del BP Koirala Institute of Health Sciences en Nepal y publicado en 2017, comparó la actividad cardiaca autónoma entre personas obesas y de peso normal a través del análisis de índices como la relación cintura-cadera e índice de masa corporal y por medio de la correlación de Spearman hicieron una relación con la variabilidad de la frecuencia cardiaca. Para ello tuvieron en cuenta medidas de baja y alta frecuencia $(0.04-0.15 \mathrm{~Hz}$ y $0.15-0.4 \mathrm{~Hz}$, respectivamente) y variables de dominio de tiempo como: SDNN, RMSSD y pNN50\%, las cuales fueron mucho menores en el grupo de personas obesas que en las de peso normal $(p<0.05)^{46}$. Entre tanto, en el estudio de Ong MEH et al. llevado a cabo en el Hospital General de Singapur, se aplicó una escala que incluía la variabilidad de la frecuencia cardiaca y los signos vitales, con el fin de observar cuál era su poder predictivo en menos de 72 horas en pacientes con dolor torácico compatible con síndrome coronario agudo. Para la variabilidad de la frecuencia cardiaca, los valores tomados fueron métodos del dominio de frecuencia: 0.01 a $0.04 \mathrm{~Hz}$ muy baja frecuencia, 0.04 a 0.15 baja frecuencia cardiaca y 0.15 a 0.4 alta frecuencia. El estudio concluye que esta escala posee mejor capacidad predictiva en menos de 72 horas en comparación con alteraciones del segmento ST en el EKG y a la alteración de las troponinas, gracias a su alta sensibilidad ${ }^{47}$.

En conclusión, puede decirse que la variabilidad de la frecuencia cardiaca es una herramienta para establecer un pronóstico temprano del desarrollo de enfermedades cardiovasculares, pero aún requiere mayor reproductibilidad en el área clínica para generar facilidades en el análisis y predecir las alteraciones de manera más oportuna. Se precisan, así mismo, estudios en nuestra comunidad teniendo en cuenta la situación epidemiológica y los recursos limitados con los que cuentan Latinoamérica, y específicamente Colombia.

\section{Conflicto de intereses}

Ninguno.

\section{Bibliografía}

1. Díaz BB, Sánchez JJA, de León AC. Frecuencia cardiaca en reposo y enfermedad cardiovascular. Med Clínica. 2014;143:34-8.

2. Lakusic N, Mahovic D, Kruzliak P, Cerkez Habek J, Novak M, Cerovec D. Changes in Heart Rate Variability after Coronary Artery Bypass Grafting and Clinical Importance of These Findings. Bio Med Res Int. 2015;2015:680515.

3. Ford CN, Slining MM, Popkin BM. Trends in dietary intake among US 2- to 6-year-old children, 1989-2008. J Acad Nutr Diet. 2013;113:35-42.

4. Sacha J. Interaction between heart rate and heart rate variability. Ann Noninvasive Electrocardiol Off J Int Soc Holter Noninvasive Electrocardiol Inc. 2014;19:207-16.

5. Achten J, Jeukendrup AE. Heart rate monitoring: applications and limitations. Sports Med Auckl NZ. 2003;33:517-38.

6. Díez B, García del MJ V, Tomás Pelegrina LJ<, Martínez JM, et al. Epidemiología de las enfermedades cardiovasculares y factores de riesgo en atención primaria. Rev Esp Cardiol. 2005;58:367-73.

7. Mariño Arévalo JJ. Correlación entre calcio coronario por tomografía y la Escala de Framingham, en estadificación del riesgo cardiovascular. Hospital José Carrasco Arteaga. Cuenca, 20162017. [Master's Thesis]. 2018.

8. Evans-Meza R, Pérez-Fallas J, Bonilla-Carrión R. Análisis de la mortalidad por enfermedades cerebrovasculares en Costa Rica entre los años 1920-2009. Arch Cardiol México. 2016;86:358-66.

9. Claveria C, Cerda Lorca J, Becker P, Schiele C, Barreno B, Urcelay $\mathrm{G}$, et al. Mortalidad operatoria y estratificación de riesgo en pacientes pediátricos operados de cardiopatía congénita: experiencia de 10 años. Rev Chil Cardiol. 2013;33:11-9.

10. Flisfisch H, Aguiló J, Cuevas IDL. Trombosis venosa profunda. Rev Med Humanidades. 2014;6:46-50.

11. Organization WH. Informe OMS sobre la epidemia mundial de tabaquismo, 2008: plan de medidas MPOWER. 2008.

12. OMS Enfermedades cardiovasculares [Internet]. WHO. [Acceso 13 Abr 2018]. Disponible en: http://www. who.int/mediacentre/factsheets/fs317/es/.

13. Kannel WB, Kannel C, Paffenbarger RS, Cupples LA. Heart rate and cardiovascular mortality: the Framingham Study. Am Heart J. 1987;113:1489-94.

14. Greenland P, Knoll MD, Stamler J, Neaton JD, Dyer AR, Garside DB, et al. Major Risk Factors as Antecedents of Fatal and Nonfatal Coronary Heart Disease Events. JAMA. 2003;290:891-7.

15. Lanas F, Avezum A, Bautista LE, Diaz R, Luna M, Islam S, et al. Risk factors for acute myocardial infarction in Latin America: the INTERHEART Latin American study. Circulation. 2007; 115:1067-74.

16. Zhu BQ, Parmley WW. Hemodynamic and vascular effects of active and passive smoking. Am Heart J. 1995;130:1270-5. 
17. Pagidipati NJ, Navar AM, Mulder H, Sniderman AD, Peterson ED, Pencina MJ. Comparison of Recommended Eligibility for Primary Prevention Statin Therapy Based on the US Preventive Services Task Force Recommendations vs the ACC / AHA Guidelines. JAMA. 2017;317:1563-7.

18. Weschenfelder Magrini D, Gue Martini J. Hipertensión arterial: principales factores de riesgo modificables en la estrategia salud de la familia. Enferm Glob. 2012;11:344-53.

19. Nishimura RA, Otto CM, Bonow RO, Carabello BA, Erwin JP, Fleisher LA, et al. 2017 AHA/ACC Focused Update of the 2014 AHA/ACC Guideline for the Management of Patients With Valvular Heart Disease: A Report of the American College of Cardiology/American Heart Association Task Force on Clinical Practice Guidelines. Circulation. 2017;135: e1159-95.

20. Padwal R, Straus SE, McAlister FA. Evidence based management of hypertension Cardiovascular risk factors and their effects on the decision to treat hypertension: evidence based review. BMJ. 2001;322:977-80.

21. Ragueneau I, Michaud P, Démolis JL, Moryusef A, Jaillon P, Funck-Brentano C. Effects of cigarette smoking on short-term variability of blood pressure in smoking and non smoking healthy volunteers. Fundam Clin Pharmacol. 1999;13:501-7.

22. Ballesteros Arribas JM, Dal-Re Saavedra M, Pérez-Farinós N, Villar Villalba C. La estrategia para la nutrición, actividad física y prevención de la obesidad: estrategia NAOS. Rev Esp Salud Pública. 2007;81:443-9.

23. Enríquez L, Matas P. [Lipoprotein (a): physiopathology and clinical and therapeutic considerations]. Med Clin (Barc). 2001;116:746-9.

24. Yildirir A, Kabakci G, Yarali H, Aybar F, Akgul E, Bukulmez O, et al. Effects of hormone replacement therapy on heart rate variability in postmenopausal women. Ann Noninvasive Electrocardiol Off J Int Soc Holter Noninvasive Electrocardiol Inc. $2001 ; 6: 280-4$

25. Huikuri HV, Jokinen V, Syvänne M, Nieminen MS, Airaksinen KE, Ikäheimo MJ, et al. Heart rate variability and progression of coronary atherosclerosis. Arterioscler Thromb Vasc Biol. 1999;19:1979-85.

26. Figueroa A, Baynard T, Fernhall B, Carhart R, Kanaley JA. Endurance training improves post-exercise cardiac autonomic modulation in obese women with and without type 2 diabetes. Eur J Appl Physiol. 2007;100:437-44.

27. de Boer RA, Nayor M, deFilippi CR, Enserro D, Bhambhani V, Kizer JR, et al. Association of cardiovascular biomarkers with incident heart failure with preserved and reduced ejection fraction. JAMA Cardiol. 2018.

28. Torres BDLC, López CL, Orellana JN. Analysis of heart rate variability at rest and during aerobic exercise. A study in healthy people and cardiac patients. Br J Sports Med. 2008.

29. Gámiz P, Luis J, Arribas Jiménez A, González Juanatey JR, Marín Huerta E, Simarro Martín-Ambrosio E. Guías de práctica clínica de la Sociedad Española de Cardiología en la monitorización ambulatoria del electrocardiograma y presión arterial. Rev Esp Cardiol. 2000;53:91-109.

30. Richards DA, Prichard BN, Hernández R. Circulatory effects of noradrenaline and adrenaline before and after labetalol. $\mathrm{Br} \mathrm{J}$ Clin Pharmacol. 1979;7:371-8.
31. Sacha J, Sobon J, Sacha K, Barabach S. Heart rate impact on the reproducibility of heart rate variability analysis. Int J Cardiol. 2013;168:4257-9.

32. Korzick DH. From syncitium to regulated pump: a cardiac muscle cellular update. Adv Physiol Educ. 2011;35:22-7.

33. Yamada R. [Catecholamines]. Horumon To Rinsho. 1971;19:838-41.

34. Miller SB. Parasympathetic nervous system control of heart rate responses to stress in offspring of hypertensives. Psychophysiology. 1994;31:11-6.

35. Haga K, Kruse AC, Asada H, Yurugi-Kobayashi T, Shiroishi M, Zhang $C$, et al. Structure of the human M2 muscarinic acetylcholine receptor bound to an antagonist. Nature. 2012;482:547-51.

36. Font GR, Pedret C, Ramos J, Ortís LC. Variabilidad de la frecuencia cardiaca: conceptos, medidas y relación con aspectos clínicos (parte II). Arch Med Deporte Rev Fed Esp Med Deporte Confed Iberoam Med Deporte. 2008:119-27.

37. Bartels R, Neumamm L, Peçanha T, Carvalho ARS. SinusCor: an advanced tool for heart rate variability analysis. Biomed Eng Online. 2017;16:110.

38. Gisselman AS, D'Amico M, Smoliga JM. Optimizing inter-session reliability of heart rate variability - the effects of artefact correction and breathing type. J Strength Cond Res. 2017.

39. Perrotta AS, Jeklin AT, Hives BA, Meanwell LE, Warburton DER. Validity of the Elite HRV Smartphone Application for Examining Heart Rate Variability in a Field-Based Setting. J Strength Cond Res. 2017;31:2296-302.

40. García-Ortiz L, García-García A, Recio-Rodríguez JI, RodríguezSánchez E, Agudo-Conde C, Gómez-Marcos MA. Variabilidad de la frecuencia cardiaca de 24 horas y lesión vascular aterosclerótica. Clínica E Investig En Arterioscler. 188-95.

41. Schroeder EB, Liao D, Chambless LE, Prineas RJ, Evans GW, Heiss G. Hypertension, blood pressure, and heart rate variability: the Atherosclerosis Risk in Communities (ARIC) study. Hypertens Dallas Tex. 1979 2003;42:1106-11.

42. Woo MA, Stevenson WG, Moser DK, Trelease RB, Harper RM. Patterns of beat-to-beat heart rate variability in advanced heart failure. Am Heart J. 1992;123:704-10.

43. Ortís LC, Font GR, Mariné MO, Romero EP, Bassets MP, Herreros MV. Variabilidad de la frecuencia cardíaca como indicador de salud en el deporte: validación con un cuestionario de calidad de vida (SF-12). Apunts Med Esport. 2008;43:62-9.

44. Berntson GG, Bigger JT, Eckberg DL, Grossman P, Kaufmann PG, Malik $M$, et al. Heart rate variability: origins, methods, and interpretive caveats. Psychophysiology. n. 1997;34:623-48.

45. Rashba EJ, Estes NAM, Wang P, Schaechter A, Howard A, Zareba $\mathrm{W}$, et al. Preserved heart rate variability identifies low-risk patients with nonischemic dilated cardiomyopathy: results from the DEFINITE trial. Heart Rhythm. 2006;3:281-6.

46. Yadav RL, Yadav PK, Yadav LK, Agrawal K, Sah SK, Islam MN. Association between obesity and heart rate variability indices: an intuition toward cardiac autonomic alteration \&ndash; a risk of CVD. Diabetes Metab Syndr Obes Targets Ther. 2017;10:57-64.

47. Ong MEH, Goh K, Fook-Chong S, Haaland B, Wai KL, Koh ZX, et al. Heart rate variability risk score for prediction of acute cardiac complications in ED patients with chest pain. Am J Emerg Med. 2013;31:7-1201. 